\title{
On the Stabilization of the Inversion of Some Kontorovich-Lebedev Like Integral Transforms
}

\author{
H.-J. Glaeske and S. B. Yakubovich
}

\begin{abstract}
In this paper we construct a special type of regularization operators for the Kontorovich-Lebedev type integral transforms to stabilize their inversion in weighted $L_{\nu, p}$-spaces. Some estimates of norms in these spaces are obtained.
\end{abstract}

Keywords: Kontorovich-Lebedev transform, Lebedev-Skalskaya transforms, index transforms, Macdonald function

AMS subject classiflcation: 44 A 15

\section{Introduction}

Following ideas of regularization for ill-posed problems, especially in the theory of integral equations, we will show that the inversion formulas of certain integral transforms can be used to stabilize the original in the sense that small changes of the transforms lead to small changes of the originals. More precisely: If the transform $g$ with

$$
g(x)=T[f](x)=\int_{0}^{\infty} H(x, y) f(y) d y
$$

is substituted by a perturbed function $h$, not necessarily in the range of $T$, and if one defines a regularization operator $I$ by means of a slight modification of the inversion operator $T^{-1}$ with

$$
T^{-1}[g](x)=f(x),
$$

one can find stability estimates with respect to $f=T^{-1}[g]$ and $I[h]$. In this sense the notion "stabilization" is used.

Our main goal is to consider a variety of the Kontorovich-Lebedev type integral transformations being connected by composition structure and mapping properties with

H.-J. Glaeske: Friedrich Schiller Univ., Inst. Math., Ernst-Abbe-PI. 1-4, D - 07743 Jena

S. B. Yakubovich: Belarusian State Univ., Dept. Math. and Mech., P.O. Box 385, 220050 Minsk, Belarus. The work was completed during this author's research visit (supported by Deutscher Akademischer Austauschdienst) to Jena University in July and August 1996 (Ref. N 325). 
the Kontorovich-Lebedev transform $[5,13,15-16]$

$$
\mathbf{K}_{i x}[f](x)=\cosh (\pi x) \int_{0}^{\infty} K_{i x}(y) f(y) d y=g(x) \quad(x \geq 0)
$$

where $K_{i x}$ is the Macdonald function (see [1: Vol.2]) and the Mehler-Fock transform [2, $4,10,16]$

$$
\mathbf{P}_{i x}[f](x)=\int_{0}^{\infty} P_{-\frac{1}{2}+i x}(\cosh y) f(y) d y=g(x)
$$

with the spherical Legendre function of the first kind $P_{-\frac{1}{2}+i x}(\cosh \cdot)($ see [1: Vol. 2]).

In Section 2 we deal with the Kontorovich-Lebedev transform (3) in the weighted $L_{p}$-space $L_{\nu, p}\left(\mathbb{R}_{+}\right)(1 \leq p \leq \infty, \nu \in \mathbb{R})$ normed by

$$
\|f\|_{\nu, p}=\left(\int_{0}^{\infty} x^{\nu p-1}|f(x)|^{p} d x\right)^{1 / p}
$$

Related problems were considered in $[3,8-12,14]$ and in two monographs [15 - 16]. Generalizing our results from the mentioned items we give a special construction of the inversion of the Kontorovich-Lebedev transform (3) that gives a solution of equation (3) and allows to stabilize it in the space $L_{\nu, p}\left(\mathbb{R}_{+}\right)$, when $\nu=1+\frac{1}{p}, 1<p<2$.

Finally, in Section 3 we will give such a construction of the inversion formula concerning the Lebedev-Skalskaya transformation [6] being related to the Kontorovich-Lebedev transform (see [15 - 16]).

\section{The Kontorovich-Lebedev transform}

Let us consider the inversion of the Kontorovich-Lebedev transform (3) as the equation where $f \in L_{1+\frac{1}{p}, p}\left(\mathbb{R}_{+}\right)$and suppose that instead of the exact function $g$ on the righthand side we have a perturbed one $h \in L_{1+\frac{1}{p}, p}\left(\mathbb{R}_{+}\right)$, not necessarily in the range of the operator $\mathbf{K}_{i x}$. Let

$$
\|h-g\|_{1+\frac{1}{p}, p}<\varepsilon
$$

for some $\varepsilon>0$. From the uniform estimate (see [16: Formula $(1.100)$ with $\delta=0]$ )

$$
\left|K_{i x}(y)\right| \leq K_{0}(y)
$$

where $K_{0}$ is the Macdonald function of zero index, and the asymptotic formulas (see [1: Vol. 2/ Subsection 7.2.2, Formula (12), resp. Subsection 7.13.1, Formula (7)])

$$
K_{i x}(y)=o(\log y) \quad(y \rightarrow 0+)
$$


and

$$
K_{i x}(y)=O\left(e^{-y} \sqrt{\pi /(2 y)}\right) \quad(y \rightarrow \infty)
$$

we observe that the integral (3) by the Holder inequality converges at infinity for all values of $x \geq 0$ and $p \geq 1$. To guarantee the convergence of the integral in (3) at zero for all values of $p \geq 1$ according to (8) we restrict the class of solutions $f$ by the additional condition $\tilde{f} \in L_{p}([0,1])$, where $\tilde{f}$ denotes the restriction of $f$ to the interval $[0,1]$. In the sequel we simply write $f \in L_{p}([0,1])$ for this.

Let us introduce a regularization operator

$$
\left(I_{\delta} h\right)(x)=\frac{2}{\pi^{2} x} \int_{0}^{\infty} \frac{\tau \sinh ((\pi-\delta) \tau)}{\cosh (\pi \tau)} K_{i \tau}(x) h(\tau) d \tau
$$

where $x>0$ and $\delta \in\left(0, \frac{\pi}{2}\right)$. Assuming that $h \in L_{1+\frac{1}{p}, p}\left(\mathbb{R}_{+}\right)$let us estimate the norm of the operator $I_{\delta}$ in this space. By means of the generalized Minkowski inequality, namely

$$
\left(\int_{a}^{b}\left|\int_{c}^{d} f(x, y) d y\right|^{p} d x\right)^{1 / p} \leq \int_{c}^{d}\left(\int_{a}^{b}|f(x, y)|^{p} d x\right)^{1 / p} d y
$$

(see [16: Formula $(1.10)]$ ), we obtain

$$
\left\|\left(I_{\delta} h\right)\right\|_{1+\frac{1}{p}, p} \leq \frac{2}{\pi^{2}} \int_{0}^{\infty} \frac{\tau \sinh ((\pi-\delta) \tau)}{\cosh (\pi \tau)}|h(\tau)|\left(\int_{0}^{\infty}\left|K_{i r}(x)\right|^{p} d x\right)^{1 / p} d \tau
$$

Hence by the Hölder inequality and estimate (7) we continue $\left(q=\frac{p}{p-1}\right)$

$$
\left\|\left(I_{\delta} h\right)\right\|_{1+\frac{1}{p}, p} \leq \frac{2}{\pi^{2}}\left(\int_{0}^{\infty}\left|K_{0}(x)\right|^{p} d x\right)^{1 / p}\|h\|_{1+\frac{1}{p}, p}\left(\int_{0}^{\infty} \frac{\sinh ^{q}((\pi-\delta) \tau)}{\cosh ^{q}(\pi \tau)} d \tau\right)^{1 / q}
$$

Estimating the integrals in (12) as

$$
\begin{aligned}
\left(\int_{0}^{\infty}\left|K_{0}(x)\right|^{p} d x\right)^{1 / p} & =\left(\int_{0}^{\infty}\left(\int_{0}^{\infty} e^{-x \cosh y} d y\right)^{p} d x\right)^{1 / p} \\
& \leq \frac{1}{p^{1 / p}} \int_{0}^{\infty} \frac{d y}{\cosh ^{1 / p} y} \\
& =\frac{2^{1 / p-2}}{p^{1 / p}} \frac{\left[\Gamma\left(\frac{1}{2 p}\right)\right]^{2}}{\Gamma\left(\frac{1}{p}\right)}
\end{aligned}
$$


and

$$
\left(\int_{0}^{\infty} \frac{\sinh ^{q}((\pi-\delta) \tau)}{\cosh ^{q}(\pi \tau)} d \tau\right)^{1 / q} \leq\left(\int_{0}^{\infty} e^{-q \delta \tau} d \tau\right)^{1 / q}=\frac{1}{(q \delta)^{1 / q}}
$$

we have finally

$$
\left\|\left(I_{\delta} h\right)\right\|_{1+\frac{1}{p}, p} \leq \frac{2^{1 / p}}{2 \pi^{2} p^{1 / p}(q \delta)^{1 / q}} \frac{[\Gamma(1 / 2 p)]^{2}}{\Gamma(1 / p)}\|h\|_{1+\frac{1}{p}, p} .
$$

For the composition $\left(I_{\delta} \mathbf{K}_{i x}[f]\right)$ the following assertion is valid.

Lemma 1. Let $f \in L_{p}([0,1]) \cap L_{1+\frac{1}{p}, p}\left(\mathbb{R}_{+}\right)(1 \leq p \leq \infty)$. Then

$$
\left(I_{\delta} \mathbf{K}_{i \tau}[f]\right)=\frac{\sin \delta}{\pi} \int_{0}^{\infty} \frac{K_{1}\left(\left(x^{2}+y^{2}-2 x y \cos \delta\right)^{1 / 2}\right)}{\left(x^{2}+y^{2}-2 x y \cos \delta\right)^{1 / 2}} y f(y) d y \quad(x>0)
$$

where $K_{1}$ is the Macdonald function of order one.

Proof. Substituting the value of $\mathbf{K}_{i r}[f]$ by formula (3) into (10), interchanging the order of integration, and then calculating the integral with respect to $\tau$ invoking formula (2.16.51.8) in [7: Vol. 2], we arrive at the representation (16). The absolute convergence of the iterated integral for arbitrary $\delta \in\left(0, \frac{\pi}{2}\right)$ can casily be shown using the Hölder inequality in the inner integral and the uniform estimate (see [16: Formula $(1.100)])$

$$
\left|K_{i \tau}(y)\right| \leq e^{-\mu \tau} K_{0}(y \cos \mu) \quad\left(\mu \in\left[0, \frac{\pi}{2}\right)\right) .
$$

The representation (16) is proved

Denoting by $f_{\epsilon}$ the left-hand side of $(10)$, where $\varepsilon$ depends upon $\delta$ (we will give such a dependence below), we write

$$
f_{\varepsilon}-f=\left(I_{\delta}(h-g)\right)+\left(I_{\delta} g\right)-f
$$

where $g=\mathbf{K}_{i x}[f]$ is the exact right-hand side of equation (3). So, by (15) and (6),

$$
\left\|f_{\varepsilon}-f\right\|_{1+\frac{1}{p}, p} \leq \frac{2^{1 / p} \varepsilon}{2 \pi^{2} p^{1 / p}(q \delta)^{1 / q}} \frac{[\Gamma(1 / 2 p)]^{2}}{\Gamma(1 / p)}+\left\|\left(I_{\delta} g\right)-f\right\|_{1+\frac{1}{p}, p} .
$$

Our purpose now is to cstimate the norm $\left\|\left(I_{\delta} g\right)-f\right\|_{1+\frac{1}{p}, p}$. Note that our approach is valid for all $1<p<2$. Substituting $y=x(\cos \delta+t \sin \delta)$ in (16) we obtain the equality

$$
\left(I_{\delta} g\right)(x)=\frac{1}{\pi} \int_{-\cot \delta}^{\infty} \frac{R(x, t, \delta)}{t^{2}+1} f(x(\cos \delta+t \sin \delta))(\cos \delta+t \sin \delta) d t
$$

where

$$
R(x, t, \delta)=x \sin \delta\left(t^{2}+1\right)^{1 / 2} K_{1}\left(x \sin \delta\left(t^{2}+1\right)^{1 / 2}\right)
$$


Hence owing to the generalized Minkowski inequality and accounting the identity

$$
\frac{1}{\pi} \int_{-\cot \delta}^{\infty} \frac{d t}{t^{2}+1}=1-\frac{\delta}{\pi}
$$

we have the estimate

$$
\begin{aligned}
\|\left(I_{\delta} g\right)- & f \|_{1+\frac{1}{p}, p} \\
\leq & \frac{1}{\pi} \int_{-\cot \delta}^{\infty} \frac{1}{t^{2}+1} \| f(x(\cos \delta+t \sin \delta))(\cos \delta+t \sin \delta) R(x, t, \delta) \\
& -\left(1-\frac{\delta}{\pi}\right)^{-1} f(x) \|_{1+\frac{1}{p}, p} d t \\
\leq & \frac{1}{\pi} \int_{-\cot \delta}^{\infty} \frac{1}{t^{2}+1} \|[f(x(\cos \delta+t \sin \delta))(\cos \delta+t \sin \delta) \\
& \left.-\left(1-\frac{\delta}{\pi}\right)^{-1} f(x)\right] R(x, t, \delta) \|_{1+\frac{1}{p}, p} d t \\
& +\frac{1}{\pi-\delta} \int_{-\cot \delta}^{\infty} \frac{1}{t^{2}+1}\|f(x)[R(x, t, \delta)-1]\|_{1+\frac{1}{p}, p} d t
\end{aligned}
$$

We estimate now each norm under the integrals in (22). For this let us assume that $f$ has a derivative $f^{\prime} \in L_{\text {loc }}^{1}\left(\mathbb{R}_{+}\right)$such that

$$
\begin{aligned}
f(x(\cos \delta+ & t \sin \delta))(\cos \delta+t \sin \delta)-\left(1-\frac{\delta}{\pi}\right)^{-1} f(x) \\
& =\int_{1}^{\cos \delta+t \sin \delta} \frac{d}{d y}[y f(x y)] d y-\frac{\delta}{\pi-\delta} f(x) \\
& =\int_{1}^{\cos \delta+t \sin \delta}\left[f(x y)+x y f^{\prime}(x y)\right] d y-\frac{\delta}{\pi-\delta} f(x) .
\end{aligned}
$$

Further, observe that owing to the uniform inequality $x K_{1}(x) \leq 1$ for the Macdonald function from (21) we obtain immediately that $R(x, t, \delta) \leq 1$. Consequently, if $f^{\prime} \in$ $L_{2+\frac{1}{p}, p}\left(\mathbb{R}_{+}\right)$, then using the generalized Minkowski inequality we have

$$
\left\|\left[f(x(\cos \delta+t \sin \delta))(\cos \delta+t \sin \delta)-\left(1-\frac{\delta}{\pi}\right)^{-1} f(x)\right] R(x, t, \delta)\right\|_{1+\frac{1}{p}, p}
$$




$$
\begin{aligned}
& \leq\|f(x(\cos \delta+t \sin \delta))(\cos \delta+t \sin \delta)-f(x)\|_{1+\frac{1}{p}, p}+\frac{\delta}{\pi-\delta}\|f\|_{1+\frac{1}{p}, p} \\
& \leq\left[\left\|f^{\prime}\right\|_{2+\frac{1}{p}, p}+\|f\|_{1+\frac{1}{p}, p}\right]\left|\int_{1}^{\cos \delta+t \sin \delta} y^{-1-\frac{1}{p}} d y\right|+\frac{\delta}{\pi-\delta}\|f\|_{1+\frac{1}{p}, p} \\
& =p\left[\left\|f^{\prime}\right\|_{2+\frac{1}{p}, p}+\|f\|_{1+\frac{1}{p}, p}\right]\left|1-(\cos \delta+t \sin \delta)^{-1 / p}\right|+\frac{\delta}{\pi-\delta}\|f\|_{1+\frac{1}{p}, p}
\end{aligned}
$$

Now for the first integral in (22) we obtain

$$
\begin{aligned}
\frac{1}{\pi} \int_{-\cot \delta}^{\infty} \frac{1}{t^{2}+1} \|[f(x(\cos \delta+t \sin \delta))(\cos \delta+t \sin \delta) \\
\left.-\left(1-\frac{\delta}{\pi}\right)^{-1} f(x)\right] R(x, t, \delta) \|_{1+\frac{1}{p}, p} d t \\
\leq \frac{p}{\pi}\left[\left\|f^{\prime}\right\|_{2+\frac{1}{p}, p}+\|f\|_{1+\frac{1}{p}, p}\right] \\
\quad \times \int_{-\cot \delta}^{\infty} \frac{\left|1-(\cos \delta+t \sin \delta)^{-1 / p}\right|}{t^{2}+1} d t+\frac{\delta}{\pi}\|f\|_{1+\frac{1}{p}, p}
\end{aligned}
$$

The integral on the right-hand side of the inequality (25) after the substitution $e^{v}=$ $\cos \delta+t \sin \delta$ takes the form $(p>1)$

$$
I_{\delta}=\frac{1}{\pi} \int_{-\cot \delta}^{\infty} \frac{\left|1-(\cos \delta+t \sin \delta)^{-1 / p}\right|}{t^{2}+1} d t=\frac{\sin \delta}{\pi} \int_{0}^{\infty} \frac{\sinh (v / p)}{\cosh v-\cos \delta} d v
$$

Hence we continue to estimate

$$
\begin{aligned}
I_{\delta} & =\frac{\sin \delta}{\pi}\left(\int_{0}^{1}+\int_{1}^{\infty}\right) \frac{\sinh (v / p)}{\cosh v-\cos \delta} d v \\
& \leq \frac{\sin \delta}{\pi}\left(\left.\log (\cosh v-\cos \delta)\right|_{0} ^{1}+\int_{1}^{\infty} \frac{\sinh (v / p)}{\cosh v-1} d v\right) \\
& \leq \sin \delta \log \left(2^{-1} \sin _{p}^{-2} \frac{\delta}{2}\right)+\sin \delta A_{p}
\end{aligned}
$$

where

$$
A_{p}=1+\int_{1}^{\infty} \frac{\sinh (v / p)}{\cosh v-1} d v \quad(p>1)
$$


Thus we can rewrite estimate (25) as

$$
\begin{aligned}
\frac{1}{\pi} \int_{-\cot \delta}^{\infty} \frac{1}{t^{2}+1} \|[f(x(\cos \delta+t \sin \delta))(\cos \delta+t \sin \delta) \\
\left.-\left(1-\frac{\delta}{\pi}\right)^{-1} f(x)\right] R(x, t, \delta) \|_{1+\frac{1}{p}, p} d t \\
\leq p \sin \delta\left[\log \left(2^{-1} \sin ^{-2} \frac{\delta}{2}\right)+A_{p}\right]\left\|f^{\prime}\right\|_{2+\frac{1}{p}, p} \\
+\left[p \sin \delta \log \left(2^{-1} \sin ^{-2} \frac{\delta}{2}\right)+p \sin \delta A_{p}+\frac{\delta}{\pi}\right]\|f\|_{1+\frac{1}{p}, p}
\end{aligned}
$$

To estimate the norm in the second integral in (22) we represent the kernel (21) by means of the identity (see [1: Vol. 2/Subsection 7.11, Formula (21)])

$$
\frac{d}{d x}\left[x K_{1}(x)\right]=-x K_{0}(x)
$$

Therefore, owing to the limit property $x K_{1}(x) \rightarrow 1$ as $x \rightarrow 0$ we obtain the representation

$$
R(x, t, \delta)-1=-\int_{0}^{x \sin \delta\left(t^{2}+1\right)^{1 / 2}} y K_{0}(y) d y
$$

Substituting it in the norm in (22) we apply the generalized Minkowski inequality and with a simple change of variables we derive

$$
\begin{aligned}
&\|f(x)[R(x, t, \delta)-1]\|_{1+\frac{1}{p}, p} \\
& \quad=\left(\int_{0}^{\infty} x^{p}\left(\int_{0}^{x \sin \delta\left(t^{2}+1\right)^{1 / 2}} y K_{0}(y) d y\right)^{p}|f(x)|^{p} d x\right)^{1 / p} \\
& \leq \int_{0}^{\infty} y K_{0}(y)\left(\int_{\frac{p}{\sin \delta\left(8^{2}+1\right)^{1 / 2}}}^{\infty} x^{p}|f(x)|^{p}\right)^{1 / p} d y .
\end{aligned}
$$

Making use of formula (32) we return to the respective integral in (22) and estimate it as follows:

$$
\frac{1}{\pi-\delta} \int_{-\cot \delta}^{\infty} \frac{1}{t^{2}+1}\|f(x)[R(x, t, \delta)-1]\|_{1+\frac{1}{p}, p} d t
$$




$$
\begin{aligned}
& \leq \frac{1}{\pi-\delta} \int_{-\cot \delta}^{\infty} \frac{1}{t^{2}+1} \int_{0}^{\infty} y K_{0}(y)\left(\int_{\frac{x}{\sin \delta\left(t^{2}+1\right)^{1 / 2}}}^{\infty} x^{p}|f(x)|^{p} d x\right)^{1 / p} d y d t \\
& =\frac{1}{\pi-\delta} \int_{-\cot \delta}^{\infty} \int_{0}^{\infty} u K_{0}\left(u \sqrt{t^{2}+1}\right) d u\left(\int_{\frac{u}{\sin \delta}}^{\infty} x^{p}|f(x)|^{p} d x\right)^{1 / p} d t
\end{aligned}
$$

Let $0<\alpha<2-p$. Dividing the integral with respect to $u$ in (33) as

$$
\left(\int_{0}^{\delta^{\alpha}}+\int_{\delta^{\alpha}}^{\infty}\right) u K_{0}\left(u \sqrt{t^{2}+1}\right) d u
$$

we continue to estimate the norm (33) and we have

$$
\begin{aligned}
& \frac{1}{\pi-\delta} \int_{-\cot \delta}^{\infty}\left(\int_{0}^{\delta^{\alpha}}+\int_{\delta^{\alpha}}^{\infty}\right)_{0} u K_{0}\left(u \sqrt{t^{2}+1}\right) d u\left(\int_{\frac{u}{\sin \delta}}^{\infty} x^{p}|f(x)|^{p} d x\right)^{1 / p} d t \\
& \leq \frac{\|f\|_{1+\frac{1}{p}, p}}{\pi-\delta} \int_{0}^{\delta^{\alpha}} u \int_{-\cot \delta}^{\infty} K_{0}(u|t|) d t d u+\int_{0}^{\infty} u K_{0}(u) d u\left(\int_{\frac{\sigma^{\alpha}}{\sin \delta}}^{\infty} x^{p}|f(x)|^{p} d x\right)^{1 / p} .
\end{aligned}
$$

Now clearly that according to the absolute continuity of the Lebesgue integral for an arbitrary $\varepsilon>0$ there exists a number $\delta \in\left(0, \frac{\pi}{2}\right)$ such that

$$
\int_{\frac{\delta^{\alpha}}{\sin 6}}^{\infty} x^{p}|f(x)|^{p} d x \leq \varepsilon^{p}
$$

Hence invoking the values of the integrals 2.16.2.1-2 from [7: Vol.2] we obtain finally

$$
\frac{1}{\pi-\delta} \int_{-\cot \delta}^{\infty} \frac{1}{t^{2}+1}\|f(x)[R(x, t, \delta)-1]\|_{1+\frac{1}{p}, p} d t<2 \delta^{\alpha}\|f\|_{1+\frac{1}{p}, p}+\varepsilon
$$

Now combining estimates (36) and (29), from (22) we get

$$
\begin{aligned}
\|\left(I_{\delta} g\right) & -f \|_{1+\frac{1}{p}, p} \\
< & p \sin \delta\left[\log \left(2^{-1} \sin ^{-2} \frac{\delta}{2}\right)+A_{p}\right]\left\|f^{\prime}\right\|_{2+\frac{1}{p}, p}+\varepsilon \\
& +\left[p \sin \delta \log \left(2^{-1} \sin ^{-2} \frac{\delta}{2}\right)+p \sin \delta A_{p}+\frac{\delta}{\pi}+2 \delta^{\alpha}\right]\|f\|_{1+\frac{1}{p}, p}
\end{aligned}
$$

Invoking inequality (35) one can let $\delta=c_{\alpha, p} \varepsilon^{p(1-\alpha)^{-1}}$, where $c_{\alpha, p}$ is a constant. Assume also that $\|f\|_{1+\frac{1}{p}, p} \leq E_{1}$ and $\left\|f^{\prime}\right\|_{2+\frac{1}{p}, p} \leq E_{2} \quad\left(E_{1}, E_{2}>0\right)$. Then, taking into account 
our previous estimates, from (19) we obtain the desired estimate of the difference (18) as

$$
\begin{aligned}
\left\|f_{\varepsilon}-f\right\|_{1+\frac{1}{p}, p}< & \frac{2^{1 / p-1}}{\pi^{2} p^{1 / p}\left(c_{\alpha, p} q\right)^{1 / q}} \frac{[\Gamma(1 / 2 p)]^{2}}{\Gamma(1 / p)} \varepsilon^{\frac{2-p-\alpha}{1-\alpha}} \\
& +p c_{\alpha, p}\left(E_{1}+E_{2}\right) \varepsilon^{p(1-\alpha)^{-1}} \log \left(2^{-1} \sin ^{-2}\left(\frac{\varepsilon^{p(1-\alpha)^{-1}} c_{\alpha, p}}{2}\right)\right) \\
& +\varepsilon+2 E_{1} c_{\alpha, p}^{\alpha} \varepsilon^{p \alpha(1-\alpha)^{-1}}+\left(p A_{p}\left(E_{1}+E_{2}\right)+1\right) c_{\alpha, p} \varepsilon^{p(1-\alpha)^{-1}}
\end{aligned}
$$

We summarize our results in this section by the following

Theorem 1. Let $f \in L_{p}([0,1]) \cap L_{1+\frac{1}{p}, p}\left(\mathbb{R}_{+}\right)(1<p<2)$ and let $f$ possess a derivative $f^{\prime} \in L_{\text {loc }}^{1}\left(\mathbb{R}_{+}\right) \cap L_{2+\frac{1}{p}, p}\left(\mathbb{R}_{+}\right)$. Then, assuming that $\|f\|_{1+\frac{1}{p}, p} \leq E_{1}$ and $\left\|f^{\prime}\right\|_{2+\frac{1}{p}, p} \leq E_{2}\left(E_{1}, E_{2}>0\right)$, the function (10), i.e.

$$
f_{c}(x)=\frac{2}{\pi^{2} x} \int_{0}^{\infty} \frac{\tau \sinh ((\pi-\delta) \tau)}{\cosh (\pi \tau)} K_{i r}(x) h(\tau) d \tau
$$

with

$$
\delta=c_{\alpha, p} \varepsilon^{p(1-\alpha)^{-1}} \quad(0<\alpha<2-p)
$$

is a regularized solution of equation (3) such that $\left\|f_{e}-f\right\|_{1+\frac{1}{p}, p}$ satisfies estimate (38) for all $1<p<2$.

\section{The Lebedev-Skalskaya transform}

In this section we will consider a regularized solution of the Lebedev-Skalskaya integral equation (see $[6,11,13]$ )

$$
\operatorname{Re}[f](x)=\cosh (\pi x) \int_{0}^{\infty} \operatorname{Re} K_{\frac{1}{2}+i x}(y) f(y) d y=g(x) \quad(x \geq 0)
$$

where $\operatorname{Re} K_{\frac{1}{2}+i x}(y)$ is the real part of the Macdonald function with the index $\frac{1}{2}+i x$, namely

$$
\operatorname{Re} K_{\frac{1}{2}+i x}(y)=\frac{K_{\frac{1}{2}+i x}(y)+K_{\frac{1}{2}-i x}(y)}{2}
$$

The integral representation of the Macdonald function (see [1: Vol. 2, Subsection 7.12, Formula (21)])

$$
K_{\nu}(x)=\int_{0}^{\infty} e^{-x \cosh u} \cosh (\nu u) d u
$$


immediately yields to the following formula for the kernel (42):

$$
\operatorname{Re} K_{\frac{1}{2}+i x}(y)=\int_{0}^{\infty} e^{-y \cosh u} \cosh \left(\frac{u}{2}\right) \cos (u x) d u
$$

and the uniform estimate

$$
\left|\operatorname{Re} K_{\frac{1}{2}+i x}(y)\right| \leq K_{\frac{1}{2}}(y)=\sqrt{\frac{\pi}{2}} \frac{e^{-y}}{\sqrt{y}} .
$$

A more precise estimate (similar to (17)) is given, for example, in [16: Formula $(6.10,11)]$, namely

$$
\left|\operatorname{Re} K_{\frac{1}{2}+i x}(y)\right| \leq e^{-\mu x} K_{\frac{1}{2}}(y \cos \mu)=\sqrt{\frac{\pi}{2}} \frac{e^{-y \cos \mu-\mu x}}{\sqrt{y \cos \mu}} \quad\left(\mu \in\left[0, \frac{\pi}{2}\right)\right) .
$$

Assume that $f \in L_{\frac{1}{p}-\frac{1}{2}, p}([0,1]) \cap L_{p}\left(\mathbb{R}_{+}\right) \quad(1<p<2)$. Then owing to (45) and the Hölder inequality it is not difficult to observe that the integral (41) converges. Indeed, we have

$$
\begin{aligned}
& \int_{0}^{\infty}\left|\operatorname{Re} K_{\frac{1}{2}+i x}(y) f(y)\right| d y \\
& \quad \leq \int_{0}^{1} \sqrt{y} K_{\frac{1}{2}}(y) \frac{|f(y)|}{\sqrt{y}} d y+\int_{1}^{\infty} K_{\frac{1}{2}}(y)|f(y)| d y \\
& \quad \leq\left(\int_{0}^{1}\left[\sqrt{y} K_{\frac{1}{2}}(y)\right]^{q} d y\right)^{1 / q}\|f\|_{L_{\frac{1}{p}-\frac{1}{2}, p}([0,1])}+\left(\int_{1}^{\infty}\left[K_{\frac{1}{2}}(y)\right]^{q} d y\right)^{1 / q}\|f\|_{p} \\
& <\infty
\end{aligned}
$$

Let us introduce a regularization operator

$$
\left(\hat{I}_{\delta} h\right)(x)=\frac{4}{\pi^{2}} \int_{0}^{\infty} \frac{\cosh ((\pi-\delta) \tau)}{\cosh (\pi \tau)} \operatorname{Re} K_{\frac{1}{2}+i \tau}(x) h(\tau) d \tau
$$

where $x>0$ and $\delta \in\left(0, \frac{\pi}{2}\right)$. Now we estimate the norm of the operator (48) under the condition $h \in L_{p}\left(\mathbb{R}_{+}\right) \quad(1<p<2)$. In the same manner as in (11) we obtain

$$
\left\|\left(\hat{I}_{\delta} h\right)\right\|_{p} \leq \frac{4}{\pi^{2}} \int_{0}^{\infty} \frac{\cosh ((\pi-\delta) \tau)}{\cosh (\pi \tau)}|h(\tau)|\left(\int_{0}^{\infty}\left|\operatorname{Re} K_{\frac{1}{2}+i \tau}(x)\right|^{p} d x\right)^{1 / p} d \tau
$$


Hence by the Hölder inequality and estimate (45) we have

$$
\left\|\left(\hat{I}_{\delta} h\right)\right\|_{p} \leq \frac{4}{\pi^{2}}\left(\int_{0}^{\infty} K_{\frac{1}{2}}^{p}(x) d x\right)^{1 / p}\|h\|_{p}\left(\int_{0}^{\infty} \frac{\cosh ^{q}((\pi-\delta) \tau)}{\cosh ^{q}(\pi \tau)} d \tau\right)^{1 / q}
$$

Estimating the integrals in $(50)$ as

$$
\begin{aligned}
\left(\int_{0}^{\infty}\left|K_{\frac{1}{2}}(x)\right|^{p} d x\right)^{1 / p} & =\sqrt{\frac{\pi}{2}}\left(\int_{0}^{\infty} e^{-p x} x^{-p / 2} d x\right)^{1 / p} \\
& =\sqrt{\frac{\pi}{2}} p^{1 / 2-1 / p}\left[\Gamma\left(1-\frac{p}{2}\right)\right]^{1 / p}
\end{aligned}
$$

and

$$
\left(\int_{0}^{\infty} \frac{\cosh ^{q}((\pi-\delta) \tau)}{\cosh ^{q}(\pi \tau)} d \tau\right)^{1 / q} \leq 2\left(\int_{0}^{\infty} e^{-q \delta \tau} d \tau\right)^{1 / q}=\frac{2}{(q \delta)^{1 / q}}
$$

we have finally

$$
\left\|\left(\hat{I}_{\delta} h\right)\right\|_{p} \leq \frac{4}{\pi \sqrt{\pi}(q \delta)^{1 / q}} p^{1 / 2-1 / p}\left[\Gamma\left(1-\frac{p}{2}\right)\right]^{1 / p}\|h\|_{p} .
$$

As a consequence of the calculations above the following lemma concerning the composition $\left(\hat{I}_{\delta} \mathbf{R e}[f]\right)$ is valid.

Lemma 2. Let $f \in L_{\frac{1}{p}-\frac{1}{2}, p}([0,1]) \cap L_{p}\left(\mathbb{R}_{+}\right)(1 \leq p \leq \infty)$. Then

$$
\begin{aligned}
\left(\hat{I}_{\delta} \operatorname{Re}[f]\right)= & \frac{\sin (\delta / 2)}{\pi}\left[\int_{0}^{\infty} K_{0}\left(\sqrt{x^{2}+y^{2}-2 x y \cos \delta}\right) f(y) d y\right. \\
& \left.+\int_{0}^{\infty} \frac{(x+y) K_{1}\left(\sqrt{x^{2}+y^{2}-2 x y \cos \delta}\right)}{\sqrt{x^{2}+y^{2}-2 x y \cos \delta}} f(y) d y\right]
\end{aligned}
$$

Proof. Substituting the value of $\operatorname{Re}[f]$ by formula (41) into (48), changing the order of integration in the absolute convergent iterated integral for any $\delta \in\left(0, \frac{\pi}{2}\right)$ as it can be shown by using the Hölder inequality in the inner integral and the estimate (46), we obtain after the calculation of the integral with respect to $\tau$ by means of formula (2.16.55.2) in [7: Vol. 2] immediately representation (54)

As above denote by $f_{e}$ the left-hand side of (54) and write the equality

$$
f_{\varepsilon}-f=\left(\hat{I}_{\delta}(h-g)\right)+\left(\hat{I}_{\delta} g\right)-f
$$

where $g=\operatorname{Re}[f]$ is the exact right-hand side of equation (41). Assuming that $\|h-g\|_{p}<$ $\varepsilon(\varepsilon>0)$ and invoking estimate (53) we have

$$
\left\|f_{e}-f\right\|_{p} \leq \frac{4 \varepsilon}{\pi \sqrt{\pi}(q \delta)^{1 / q}} p^{1 / 2-1 / p}\left[\Gamma\left(1-\frac{p}{2}\right)\right]^{1 / p}+\left\|\left(\hat{I}_{\delta} g\right)-f\right\|_{p}
$$


To estimate the norm $\left\|\left(\hat{I}_{\delta} g\right)-f\right\|_{p}$ by the same substitution as in (20) we obtain the representation

$$
\left(\hat{I}_{\delta} g\right)(x)=\frac{1}{\pi} \int_{-\cot \delta}^{\infty} \frac{\hat{R}(x, t, \delta)}{t^{2}+1} f(x(\cos \delta+t \sin \delta)) d t
$$

where

$$
\begin{aligned}
\hat{R}(x, t, \delta)= & x \sin \frac{\delta}{2}\left[\sin \delta\left(t^{2}+1\right) K_{0}\left(x \sin \delta\left(t^{2}+1\right)^{1 / 2}\right)\right. \\
& \left.+(1+\cos \delta+t \sin \delta)\left(t^{2}+1\right)^{1 / 2} K_{1}\left(x \sin \delta\left(t^{2}+1\right)^{1 / 2}\right)\right]
\end{aligned}
$$

Hence similar as in the case of the Kontorovich-Lebedev transform we have the estimate

$$
\begin{aligned}
\|\left(\hat{I}_{\delta} g\right) & -f \|_{p} \\
\leq & \frac{1}{\pi} \int_{-\cot \delta}^{\infty} \frac{1}{t^{2}+1}\left\|f(x(\cos \delta+t \sin \delta)) \hat{R}(x, t, \delta)-\left(1-\frac{\delta}{\pi}\right)^{-1} f(x)\right\|_{p} d t \\
\leq & \frac{\sin \frac{\delta}{2} \sin \delta}{\pi} \int_{-\cot \delta}^{\infty}\left\|f(x(\cos \delta+t \sin \delta)) x K_{0}\left(x \sin \delta\left(t^{2}+1\right)^{1 / 2}\right)\right\|_{p} d t \\
& +\frac{1}{\pi} \int_{-\cot \delta}^{\infty} \frac{1}{t^{2}+1} \| f(x(\cos \delta+t \sin \delta)) x \sin \frac{\delta}{2}\left(t^{2}+1\right)^{1 / 2} \\
& \times K_{1}\left(x \sin \delta\left(t^{2}+1\right)^{1 / 2}\right)-\frac{\pi}{2(\pi-\delta)} f(x) \|_{p} d t \\
& +\frac{1}{\pi} \int_{-\cot \delta}^{\infty} \frac{1}{t^{2}+1} \| f(x(\cos \delta+t \sin \delta))(\cos \delta+\sin \delta) x \sin \frac{\delta}{2}\left(t^{2}+1\right)^{1 / 2} \\
& \times K_{1}\left(x \sin \delta\left(t^{2}+1\right)^{1 / 2}\right)-\frac{\pi}{2(\pi-\delta)} f(x) \|_{p} d t \\
= & I_{1}(\delta)+I_{2}(\delta)+I_{3}(\delta) .
\end{aligned}
$$

Let us estimate now each of the integrals $I_{i}(\delta) \quad(1 \leq i \leq 3)$. Since for all $x>0$ the inequality $x K_{0}(x)<1$ holds, then for the integral $I_{1}(\delta)$ we obtain

$$
\begin{aligned}
I_{1}(\delta) & =\frac{\sin \frac{\delta}{2} \sin \delta}{\pi} \int_{-\cot \delta}^{\infty}\left\|f(x(\cos \delta+t \sin \delta)) x K_{0}\left(x \sin \delta\left(t^{2}+1\right)^{1 / 2}\right)\right\|_{p} d t \\
& <\frac{\sin \frac{\delta}{2}\|f\|_{p}}{\pi} \int_{-\cot \delta}^{\infty} \frac{d t}{\sqrt{t^{2}+1}(\cos \delta+t \sin \delta)^{1 / p}} \\
& =\frac{\sqrt{2} \sin \frac{\delta}{2}}{\pi}\|f\|_{p} \int_{0}^{\infty} \frac{\cosh \left(\left(\frac{1}{p}-\frac{1}{2}\right) v\right)}{\sqrt{\cosh v-\cos \delta}} d v .
\end{aligned}
$$


Note that the last integral is obtained by the substitution $e^{v}=\cos \delta+t \sin \delta$. Hence we write $(1<p<2)$

$$
\begin{aligned}
& \left(\int_{0}^{1}+\int_{1}^{\infty}\right) \frac{\cosh \left(\left(\frac{1}{p}-\frac{1}{2}\right) v\right)}{\sqrt{\cosh v-\cos \delta}} d v \\
& \leq 2 \sqrt{2} \cosh \left(\frac{1}{p}-\frac{1}{2}\right) \int_{0}^{1} \frac{d v}{\sqrt{v^{2}+16 \sin ^{2}(\delta / 2)}}+\frac{1}{\sqrt{2}} \int_{1}^{\infty} \frac{\cosh \left(\left(\frac{1}{p}-\frac{1}{2}\right) v\right)}{\sinh (v / 2)} d v \\
& =B_{p} \log \left(4 \sin \left(\frac{\delta}{2}\right)+\sqrt{1+16 \sin ^{2}\left(\frac{\delta}{2}\right)}\right)+C_{p}
\end{aligned}
$$

where the constants $B_{p}$ and $C_{p}$ are defined as

$$
B_{p}=2 \sqrt{2} \cosh \left(\frac{1}{p}-\frac{1}{2}\right) \quad \text { and } \quad C_{p}=\frac{1}{\sqrt{2}} \int_{1}^{\infty} \frac{\cosh \left(\left(\frac{1}{p}-\frac{1}{2}\right) v\right)}{\sinh (v / 2)} d v
$$

Thus we obtain finally

$$
I_{1}(\delta)<\frac{\sqrt{2} \sin \frac{\delta}{2}}{\pi}\left[B_{p} \log \left(4 \sin \left(\frac{\delta}{2}\right)+\sqrt{1+16 \sin ^{2}\left(\frac{\delta}{2}\right)}\right)+C_{p}\right]\|f\|_{p}
$$

Concerning the sum of integrals $I_{2}(\delta)+I_{3}(\delta)$ similar to (22) and (24) we derive

$$
\begin{aligned}
I_{2}(\delta) & +I_{3}(\delta) \\
\leq & \frac{1}{2 \pi} \int_{-\cot \delta}^{\infty} \frac{1}{t^{2}+1}\left\|f(x(\cos \delta+t \sin \delta))-\frac{\pi}{\pi-\delta} f(x)\right\|_{p} d t \\
& +\frac{1}{2 \pi} \int_{-\cot \delta}^{\infty} \frac{1}{t^{2}+1}\left\|f(x(\cos \delta+t \sin \delta))(\cos \delta+t \sin \delta)-\frac{\pi}{\pi-\delta} f(x)\right\|_{p} d t \\
& +\frac{1}{\pi-\delta} \int_{-\cot \delta}^{\infty} \frac{1}{t^{2}+1} \\
& \times\left\|f(x)\left[2 x \sin \frac{\delta}{2}\left(t^{2}+1\right)^{1 / 2} K_{1}\left(x \sin \delta\left(t^{2}+1\right)^{1 / 2}\right)-\cos ^{-1} \frac{\delta}{2}\right]\right\|_{p} d t \\
& +\frac{2 \sin ^{2}(\delta / 4)}{\pi \cos (\delta / 2)}\|f\|_{p} .
\end{aligned}
$$

Hence, appealing to (36) for the third integral of the right-hand side of inequality (64) 
we immediately obtain the estimate

$$
\begin{aligned}
& \frac{1}{\pi-\delta} \int_{-\cot \delta}^{\infty} \frac{1}{t^{2}+1} \\
& \times\left\|f(x)\left[2 x \sin \frac{\delta}{2}\left(t^{2}+1\right)^{1 / 2} K_{1}\left(x \sin \delta\left(t^{2}+1\right)^{1 / 2}\right)-\cos ^{-1} \frac{\delta}{2}\right]\right\|_{p} d t \\
& <2 \delta^{\alpha} \cos ^{-1} \frac{\delta}{2}\|f\|_{p}+\varepsilon
\end{aligned}
$$

where for an arbitrary $\varepsilon>0$ there exists such a $\delta \in\left(0, \frac{\pi}{2}\right)$ that

$$
\int_{\frac{\delta}{\sin \delta}}^{\infty}|f(x)|^{p} d x \leq \epsilon^{p} \quad(0<\alpha<2-p)
$$

The second integral in the right-hand side of (64) is estimated by means of representation (23) under the condition that $f$ has a derivative $f^{\prime} \in L_{1+\frac{1}{p}, p}\left(\mathbb{R}_{+}\right)$. At first we have

$$
\begin{aligned}
& \frac{1}{2 \pi} \int_{-\cot \delta}^{\infty} \frac{1}{t^{2}+1}\left\|f(x(\cos \delta+t \sin \delta))(\cos \delta+t \sin \delta)-\frac{\pi}{\pi-\delta} f(x)\right\|_{p} d t \\
& \leq \frac{q}{2 \pi}\left[\left\|f^{\prime}\right\|_{1+\frac{1}{p}, p}+\|f\|_{p}\right] \int_{-\cot \delta}^{\infty} \frac{\left|1-(\cos \delta+t \sin \delta)^{1 / q}\right|}{t^{2}+1} d t+\frac{\delta}{\pi}\|f\|_{p} .
\end{aligned}
$$

The integral on the right-hand side of inequality (67) can be treated in the same manner as in (26) - (28). Thus we obtain an analogy of incquality (29), precisely

$$
\begin{aligned}
\frac{1}{2 \pi} \int_{-\cot \delta}^{\infty} \frac{1}{t^{2}+1}\left\|f(x(\cos \delta+t \sin \delta))(\cos \delta+t \sin \delta)-\frac{\pi}{\pi-\delta} f(x)\right\|_{p} d t \\
\leq \frac{q}{2} \sin \delta\left[\log \left(2^{-1} \sin ^{-2} \frac{\delta}{2}\right)+A_{q}\right]\left\|f^{\prime}\right\|_{1+\frac{1}{p}, p} \\
+\left[\frac{q}{2} \sin \delta \log \left(2^{-1} \sin ^{-2} \frac{\delta}{2}\right)+\frac{q}{2} \sin \delta A_{q}+\frac{\delta}{2 \pi}\right]\|f\|_{p}
\end{aligned}
$$

where the constant $A_{q}$ is defined by equality (28) and $q$ as usualy equals $\frac{p}{p-1}$. Let us estimate the first integral in the right-hand side of inequality (64). By using the equality

$$
f(x(\cos \delta+t \sin \delta))-\frac{\pi}{\pi-\delta} f(x)=x \int_{1}^{\cos \delta+t \sin \delta} \cdot f^{\prime}(x y) d y-\frac{\delta}{\pi-\delta} f(x)
$$


we have

$$
\begin{aligned}
& \left\|f(x(\cos \delta+t \sin \delta))-\frac{\pi}{\pi-\delta} f(x)\right\|_{p} \\
& \quad \leq p\left[\left\|f^{\prime}\right\|_{1+\frac{1}{p}, p}+\|f\|_{p}\right]\left|1-(\cos \delta+t \sin \delta)^{-1 / p}\right|+\frac{\delta}{\pi-\delta}\|f\|_{p}
\end{aligned}
$$

and consequently the final estimate will take the form

$$
\begin{aligned}
\frac{1}{2 \pi} \int_{-\cot \delta}^{\infty} \frac{1}{t^{2}+1}\left\|f(x(\cos \delta+t \sin \delta))-\frac{\pi}{\pi-\delta} f(x)\right\|_{p} d t \\
\leq \frac{p}{2} \sin \delta\left[\log \left(2^{-1} \sin ^{-2} \frac{\delta}{2}\right)+A_{p}\right]\left\|f^{\prime}\right\|_{1+\frac{1}{p}, p} \\
+\left[\frac{p}{2} \sin \delta \log \left(2^{-1} \sin ^{-2} \frac{\delta}{2}\right)+\frac{p}{2} \sin \delta A_{p}+\frac{\delta}{2 \pi}\right]\|f\|_{p} .
\end{aligned}
$$

Now meaning $\delta=\hat{c}_{\alpha, p} \varepsilon^{p(1-\alpha)^{-1}}$, where $\hat{c}_{\alpha, p}$ is a constant, and assuming that $\|f\|_{p} \leq E_{1}$ and $\left\|f^{\prime}\right\|_{1+\frac{1}{p}, p} \leq E_{2}\left(E_{1}, E_{2}>0\right)$ from (56), (59) taking into account estimates (63) (65), (68) and (71) we obtain

$$
\begin{aligned}
\| f_{\varepsilon}- & f \|_{p} \\
< & \frac{4 \varepsilon^{(2-p-\alpha) /(1-\alpha)}}{\pi \sqrt{\pi}\left(q \hat{c}_{\alpha, p}\right)^{1 / q}} p^{1 / 2-1 / p}\left[\Gamma\left(1-\frac{p}{2}\right)\right]^{1 / p} \\
& +\frac{1}{2}\left(E_{1}+E_{2}\right)(p+q) \hat{c}_{\alpha, p} \varepsilon^{p(1-\alpha)^{-1}} \log \left(2^{-1} \sin ^{-2}\left(\frac{\hat{c}_{\alpha, p}}{2} \varepsilon^{p(1-\alpha)^{-1}}\right)\right) \\
& +\frac{\sqrt{2}}{\pi} E_{1} B_{p} \sin \left(\frac{\hat{c}_{\alpha, p}}{2} \varepsilon^{p(1-\alpha)^{-1}}\right) \\
& \times \log \left(4 \sin \left(\frac{\hat{c}_{\alpha, p}}{2} \varepsilon^{p(1-\alpha)^{-1}}\right)+\sqrt{1+16 \sin ^{2}\left(\frac{\hat{c}_{\alpha, p}}{2} \varepsilon^{p(1-\alpha)^{-1}}\right)}\right) \\
& +\left(E_{1}\left(C_{p}+1\right)+\frac{1}{2}\left(p A_{p}+q A_{q}\right)\left(E_{1}+E_{2}\right)\right) \hat{c}_{\alpha, p} \varepsilon^{p(1-\alpha)^{-1}}+\varepsilon \\
& +E_{1}\left(\frac{2 \sin ^{2}\left(\hat{c}_{\alpha, p} \varepsilon^{p(1-\alpha)^{-1}} / 4\right)}{\pi \cos \left(\hat{c}_{\alpha, p} \varepsilon^{\left.p(1-\alpha)^{-1} / 2\right)}\right.}+2 \hat{c}_{\alpha, p}^{\alpha} \varepsilon^{p \alpha(1-\alpha)^{-1}} \cos ^{-1}\left(\frac{\hat{c}_{\alpha, p}}{2} \varepsilon^{p(1-\alpha)^{-1}}\right)\right) .
\end{aligned}
$$

Therefore we have proved the following

Theorem 2. Let $f \in L_{\frac{1}{p}-\frac{1}{2}, p}([0,1]) \cap L_{1+\frac{1}{p}, p}\left(\mathbb{R}_{+}\right) \quad(1<p<2)$ and let $f$ possess a derivative $f^{\prime} \in L_{\text {loc }}^{1}\left(\mathbb{R}_{+}\right) \cap L_{1+\frac{1}{p}, p}\left(\mathbb{R}_{+}\right)$. Then assuming that $\|f\|_{p} \leq E_{1}$ and $\left\|f^{\prime}\right\|_{1+\frac{1}{p}, p} \leq$ $E_{2} \quad\left(E_{1}, E_{2}>0\right)$ the function (48), i.e.

$$
f_{e}(x)=\frac{4}{\pi^{2}} \int_{0}^{\infty} \frac{\cosh ((\pi-\delta) \tau)}{\cosh (\pi \tau)} \operatorname{Re} K_{\frac{1}{2}+i r}(x) h(\tau) d \tau
$$


with

$$
\delta=\hat{c}_{\alpha, p} \varepsilon^{p(1-\alpha)^{-1}} \quad(0<\alpha<2-p)
$$

is a regularized solution of equation (41) such that $\left\|f_{e}-f\right\|_{p}$ satisfies estimate (72) for all $1<p<2$.

\section{References}

[1] Eedelyi, A., Magnus, W., Oberhettinger, F. and F. G. Tricomi: Higher Transcendental Functions. Vols I and II. New York: McGraw-Hill 1953.

[2] Glaeske, H.-J.: Some investigations concerning the Mehler-Fock and the KontorovichLebedev transformations. Proc. Intern. Conf. Complex Anal. and Appl., Varna, 1985, ed.: L. Iliev, I. Ramadanov, T. Tonev. Sofia: Publ. House Bulg. Acad. Sci. 1986, pp. $228-238$.

[3] Glaeske, H.-J. and A. Hess: A convolution connected with the Kontorovich-Lebedev transform. Math. Z. 193 (1986), $67-78$.

[4] Glaeske, H.-J. and A. Hess: On the convolution theorem of the Mehler-Fock-Transform for a class of generalized functions. Part I. Math. Nachr. 131 (1987), $107-117$.

[5] Lebedev, N. N.: Sur une formule d'inversion. C.R. (Dokl.) Acad. Sci. SSR 52 (1946), $655-658$.

[6] Lebedev, N. N. and I. P. Skalskaya: Some integral transforms related to the KontorovichLebedev transform (in Russian). In: The Questions of Math. Physics. Leningrad: Nauka 1976, pp. 68 - 79.

17) Prudnikov, A. P., Brychkov, Yu. A. and O. I. Marichev: Integrals and Series. Vols. 1 - 3. New York - London: Gordon and Breach 1986, 1986 and 1989.

[8] Yakubovich, S. B.: A general approach to the theory of integral transforms with respect to an index (in Russian). Izv. Vuzov, Matematika 6 (1986), 77 - 79.

[9] Yakubovich, S. B.: The Kontorovich-Lebedev type transforms and their convolutions. In: Proc. Intern. Conf. Complex Anal. and Appl., with Symp. on Gen. Functions, Varna 1991. ed.: I. Dimovski, V. Hristov. Sofia: Publ. House Bulg. Acad. Sci. 1993, pp. 348 360 .

[10] Yakubovich, S. B.: On the Mehler-Fock integral transform in $L_{p}$-spaces. Extracta Math. 8 (1993), $162-164$.

[11] Yakubovich, S. B.: On the construction of integral transforms by the composition method (in Russian). Izv. Vuzov., Matematika 9 (1993), $71-79$.

[12] Yakubovich, S. B.: Some compositions theorems of Plancherel type for index transforms (in Russian). Dokl. Akad. Nauk Belarusi 38 (1994), $29-32$.

[13] Yakubovich, S. B.: On some new properties of the Kontorovich-Lebedev like integral transforms. Rev. Tec. Ing. Univ. Zulia 18 (1995), $291-299$.

[14] Yakubovich, S. B.: On the new approach to constructions of the index transforms. Diss. Math. 340 (1995), 321 - 335.

[15] Yakubovich, S. B.: Index Transforms. Singarpore etc.: World Sci. 1996.

[16] Yakubovich, S: B. and Yu. F. Luchko: The Hypergeometric Approach to Integral Transforms and Convolutions (Kluwers Ser. Math. and Appl.: Vol. 287). Dordrecht - Boston - London: Kluwer 1994. 